\title{
Cytoprotective effects of Lycium barbarum against reducing stress on endoplasmic reticulum
}

\author{
MAN-SHAN YU ${ }^{1}$, YUEN-SHAN HO ${ }^{1}$, KWOK-FAI SO ${ }^{1-3}$, \\ WAI-HUNG YUEN ${ }^{4}$ and RAYMOND CHUEN-CHUNG CHANG ${ }^{1-3}$ \\ ${ }^{1}$ Laboratory of Neurodegenerative Diseases, Department of Anatomy, ${ }^{2}$ Research Centre of Heart, \\ Brain, Hormone and Healthy Aging, Faculty of Medicine, ${ }^{3}$ Central Laboratory of the Institute \\ of Molecular Technology for Drug Discovery and Synthesis, ${ }^{4}$ Department of Chemistry \\ and Open Laboratory of Chemical Biology of the Institute of Molecular Technology for \\ Drug Discovery and Synthesis, The University of Hong Kong, Hong Kong, SAR
}

Received December 1, 2005; Accepted January 23, 2006

\begin{abstract}
Chinese medicinal herbs have been consumed for thousands of years for the purpose of healthy aging. Lycium barbarum is valued in Chinese culture for its benefits to antiaging, vision, kidney and liver. Recent studies showed that extracts from L. barbarum possess biological activities including anti-aging, anti-tumor, immune-stimulatory and cytoprotection. Most of these studies emphasized that the protective function of L. barbarum is due to its anti-oxidative effects. We have previously demonstrated that extract from $L$. barbarum can protect neurons against $\beta$-amyloid (Aß) peptide-induced apoptosis. Since Aß toxicity may be mediated via oxidative stress, it is still unclear whether the extract from L. barbarum is a simple anti-oxidant exhibiting cytoprotective effects. We hypothesized that extract from L. barbarum is not simply an anti-oxidant in order to function as a neuroprotective agent. The aim of this study is to investigate whether the extract from L. barbarum (LBG) protect neurons via mechanisms independent of anti-oxidative effects. Using a reducing agent, dithiothreitol (DTT), we found that LBG exhibits cytoprotective effects against reducing stress by lowering the DTT-induced LDH release and caspase-3 activity. DTT can trigger endoplasmic reticulum (ER) stress leading to PKR-like ER kinase (PERK) activation. We also showed that LBG attenuates DTT-induced PERK phosphorylation. The extract from L. barbarum is not simply an anti-oxidant; it can also exhibit cytoprotective effects against reducing stress by DTT.
\end{abstract}

Correspondence to: Dr Raymond Chuen-Chung Chang, Department of Anatomy, Faculty of Medicine, The University of Hong Kong, 21 Sassoon Road, Hong Kong, SAR

E-mail: rccchang@hkucc.hku.hk

Key words: Lycium barbarum, cytoprotective effects, reducing stress, endoplasmic reticulum

\section{Introduction}

The fruits of Lycium barbarum (also called Fructus Lycii, Wolfberry or Gouqizi), the small red berries, have been used for thousands of years in traditional Chinese medicine and cuisine since L. barbarum is believed to be beneficial to the eyes, liver and kidney. Different biological activities of $L$. barbarum have been demonstrated, including anti-aging, antitumor, immune-stimulatory and cytoprotection (1-3). Many studies suggested that the protective effect of $L$. barbarum mainly depends on its anti-oxidative action. For example, $L$. barbarum polysaccharides can capture free radicals and restrain the DNA damage of testicle cells caused by the oxidative stress, $\mathrm{H}_{2} \mathrm{O}_{2}$ (4). Another report showed that Fructus Lycii polysaccharides protect cultured seminiferous epithelium against hyperthermia-induced damage via an anti-oxidant mechanism (5). We have previously proved that the aqueous extract of $L$. barbarum exhibits neuroprotective effects against $\beta$-amyloid $(A B)$ peptide-induced apoptosis in cultured neurons (6). Generation of reactive oxygen species (ROS) may be one of the mechanisms caused by $A B$ to trigger neuronal cell death (7). Thus, we cannot exclude the possibility that the neuroprotective effects of the aqueous extract of L. barbarum against $A ß$ toxicity are mediated through anti-oxidation. It is an interesting question to ask whether the extract from $L$. barbarum is simply an anti-oxidant. We hypothesized that anti-oxidative effects are not the sole neuroprotective effects exhibited by L. barbarum. The aim of this study is to investigate whether the extract from L. barbarum (LBG) protects neurons via mechanisms independent of anti-oxidation. Dithiothreitol (DTT), an ER stress inducer, was used to trigger reducing stress in neurons by inhibiting the formation of disulfide bonds in the ER $(8,9)$. We found that LBG exhibited cytoprotective effects against this reducing stress by lowering the DTT-induced LDH and caspase-3 activity. LBG also attenuated DTT-induced PERK phosphorylation. Therefore, the extract from L. barbarum is not simply an anti-oxidant; it can also exhibit cytoprotective effects against reducing stress on the ER. 


\section{Materials and methods}

Materials. Lycium barbarum extract (LBG) from The Hong Kong Institute of Biotechnology (Shatin, Hong Kong) was used in this study. LBG was standardized by their chemical composition using thin layer chromatography to ensure batch-to-batch consistency. Materials used for neuronal cell culture were purchased from Gibco BRL (Burlington, Ontario, Canada). 1,4-Dithiothreitol (DTT), all-trans retinoic acid (RA), protease inhibitor cocktail, phosphatase inhibitor cocktail and anti- $\beta$-actin monoclonal antibody were obtained from SigmaAldrich, Inc. (St. Louis, MO, USA); the LDH cytotoxicity assay kit was from Roche Diagnostics (Mannheim, Germany); colorimetric caspase-3 substrate (Ac-DEVD-pNA) was from Calbiochem, Inc. (Darmstadt, Germany); rabbit polyclonal antibodies for phospho-PERK were from Cell Signaling Technology (Beverly, MA, USA); horseradish peroxidase (HRP)-conjugated goat anti-rabbit and goat anti-mouse antibodies were from Dako (Glostrup, Denmark); PVDF membrane was from Bio-Rad (Richmond, CA, USA); Biomax $\mathrm{X}$-way film was from Kodak (Tokyo, Japan); the enhanced chemiluminescence (ECL) detection kit was from Amersham (Buckinghamshire, UK); and the Re-Blot Western blot recycling kit was from Chemicon International, Inc. (Temecula, CA, USA).

Primary neuronal cultures and human neuroblastoma cell line. Primary neuronal cultures were prepared from embryonic day 17 Sprague-Drawley rats (Laboratory Animal Unit, The University of Hong Kong) according to our previous published methods (6,10-15). Cortical neurons were cultured for 7 days with minimum essential medium (MEM) supplemented with $5 \%$ fetal bovine serum and antibiotics prior to the treatment. Human neuroblastoma SH-SY5Y cells were obtained from American Tissue Type Collection (ATTC, Rockville, MD, USA). SH-SY5Y cells were cultured with MEM supplemented with $10 \%$ fetal bovine serum, $1 \%$ glutamine, penicillin (50 U/ $\mathrm{ml})$ and streptomycin $(50 \mu \mathrm{g} / \mathrm{ml})$. The cells were differentiated with medium containing $3 \%$ fetal bovine serum and $10 \mu \mathrm{M}$ all-trans retinoic acid for 7 days before the treatment.

Treatment of neurons. In order to investigate whether LBG exerts neuroprotective effects, the cells were treated with LBG at concentrations ranging from $10 \mu \mathrm{g} / \mathrm{ml}$ to $500 \mu \mathrm{g} / \mathrm{ml}$ for $1 \mathrm{~h}$. Then, cultured neurons were exposed to DTT (1 mM) for $24 \mathrm{~h}$ as our previous experiments showed that this treatment condition could induce ER stress (15). The culture medium and cells were harvested for different biological assays.

Measurement of general cytotoxicity. Culture medium was collected for LDH activity assay to determine the level of general cytotoxity. The procedures were carried out in accordance with the manufacturer's instructions and our previous published method $(6,11,12)$. In brief, the culture medium was incubated with assay reagents in the dark for $30 \mathrm{~min}$. The absorbance at $492 \mathrm{~nm}$ was measured to determine the level of LDH released. The results were expressed as the fold of control.

Caspase-3-like activity assay. Neurons were harvested for protein extraction, protein assay and caspase-3-like activity (a)

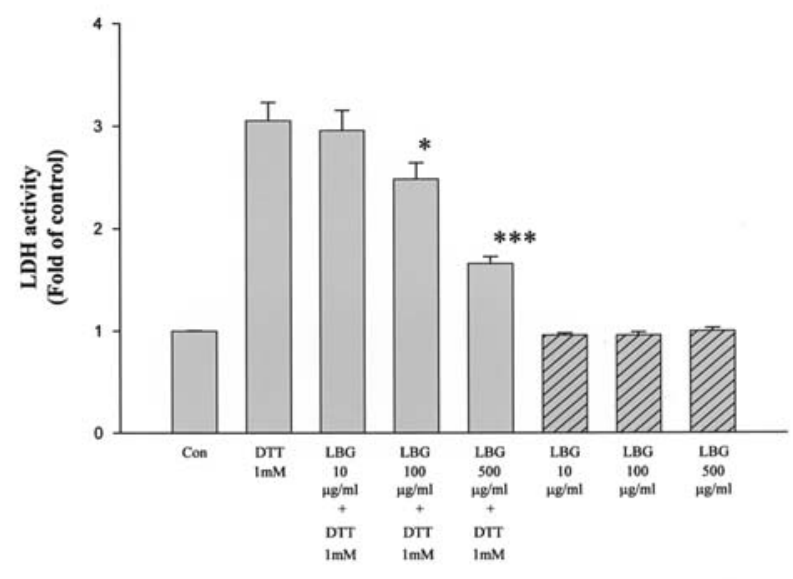

(b)

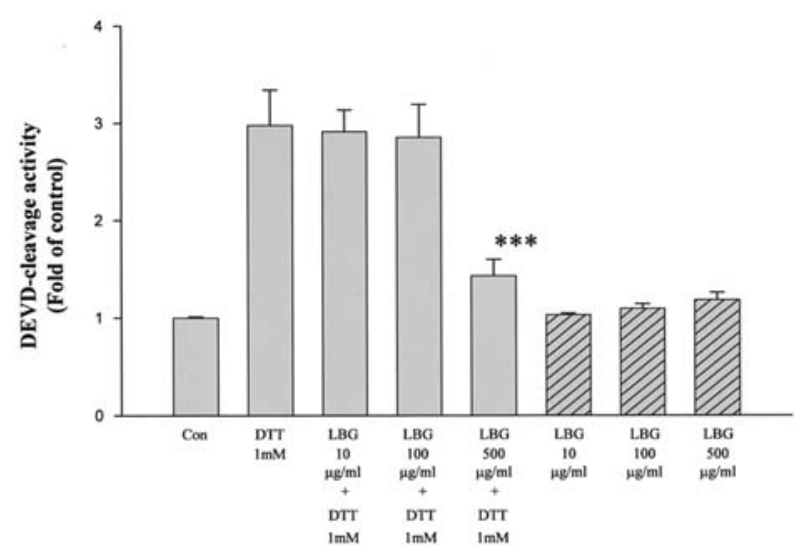

Figure 1. Neuroprotective effects of the LBG extract on DTT-induced cytotoxicity in cultured rat cortical neurons. Cortical neurons prepared from embryonic SD rats were treated with different concentrations of LBG for $1 \mathrm{~h}$, followed by a 24-h incubation with or without DTT (1 mM). (a) LDH activity assay was performed to measure the level of cytotoxicity. LDH activity was expressed as fold of control. (b) Caspase-3 activity assay was performed to determine the extent of neuronal apoptosis. DEVD-cleavage activity was expressed as fold of control. Results are expressed as mean $\pm \mathrm{SE}$ from at least three independent experiments. ${ }^{* * *} \mathrm{p}<0.001$ and ${ }^{*} \mathrm{p}<0.05$ vs. the group treated with DTT only by one-way ANOVA for multiple comparison and Student-Newman-Keuls test as post-hoc test.

assay as described in our previous studies $(6,10-17)$. In principle, colorimetric caspase-3 substrate (Ac-DEVD-pNA) was cleaved to form a yellow product (pNA). The absorbance of the yellow product at $405 \mathrm{~nm}$ was measured to determine the caspase-3-like activity. The values of specific activity (s.a., unit $=\mathrm{pmol} / \mathrm{min} / \mu \mathrm{g}$ ) were calculated from the absorbance reading. Results were expressed as the fold of control.

Western blotting. To examine the level of phospho-PERK, primary cultured neurons after different treatments were harvested for protein extraction and protein assay as described in our previous studies $(6,10-17)$. Fifty micrograms of protein per lane were separated in sodium dodecyl sulphate-polyacrylamide gel electrophoresis (SDS-PAGE). Proteins were then transferred onto a polyvinylidene difluoride (PVDF) membrane. The membrane was incubated with anti-phosphoPERK antibody (1:1000) for $2 \mathrm{~h}$ at room temperature, followed by incubation with goat anti-rabbit HRP-conjugated 

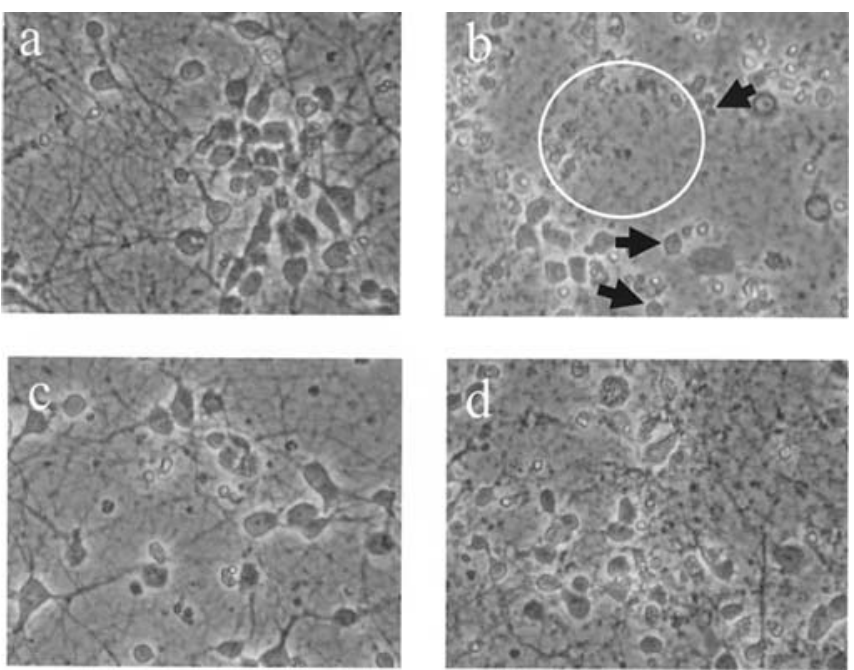

Figure 2. Morphology of cultured neurons after different treatments. Neurons were pretreated with LBG for $1 \mathrm{~h}$, followed by a 24-h exposure to DTT. (a) Control, (b) DTT (1 mM) (arrow indicates shrunk cell body and the circled area indicates the destructed neurite network), (c) LBG (500 $\mu \mathrm{g} / \mathrm{ml})$ and (d) LBG $(500 \mu \mathrm{g} / \mathrm{ml})+$ DTT $(1 \mathrm{mM})$

secondary antibodies $(1: 2000)$ for $1 \mathrm{~h}$ at room temperature. Bands on X-ray film were developed by using a chemiluminescent ECL detection kit. Membranes were then stripped using the Re-Blot Western blot recycling kit and re-probed with anti- $\beta$-actin antibody (1:5000) as primary antibody, and goat anti-mouse-HRP as secondary antibody.

Statistical analysis. For quantitative data, the results are expressed as mean \pm SE from at least three independent experiments. One-way ANOVA was used to determine the significance of differences among different groups, followed by Student-Newman-Keuls test as post-hoc test. A value of $\mathrm{p}<0.001$ or $\mathrm{p}<0.05$ was considered statistically significant in all experiments.

\section{Results}

Neuroprotective effects of the LBG extract in cultured rat cortical neurons. To examine the neuroprotective effects of the LBG extract on DTT-induced cell death, cortical neurons prepared from embryonic SD rats were pre-treated with LBG at 10,100 or $500 \mu \mathrm{g} / \mathrm{ml}$ for $1 \mathrm{~h}$, followed by incubation with or without the reducing stress DTT $(1 \mathrm{mM})$ for $24 \mathrm{~h}$. After treatment, the extent of general cytotoxicity and apoptosis was determined by LDH and caspase-3 activity assays, respectively. Fig. 1a shows the LDH activity of various treatment groups. For the control group, LDH activity was calculated as $1.00 \pm 0.00$ fold of control, neurons treated only with DTT showed a significant increase in LDH activity to $3.05 \pm 0.18$-fold of control. Pretreatment with LBG at 100 and $500 \mu \mathrm{g} / \mathrm{ml}$ markedly reduced the LDH release triggered by DTT $(2.48 \pm 0.16$ and $1.66 \pm 0.07$-fold of control). To examine the effects of LBG on DTT-activated caspase-3 activity, the colorimetric caspase-3like activity assay was performed. In Fig. 1b, the DEVDcleavage was $2.98 \pm 0.36$-fold of control of the group treated with DTT only. LBG at $500 \mu \mathrm{g} / \mathrm{ml}$ showed the best neuroprotective effect since it lowered the DEVD-cleavage to

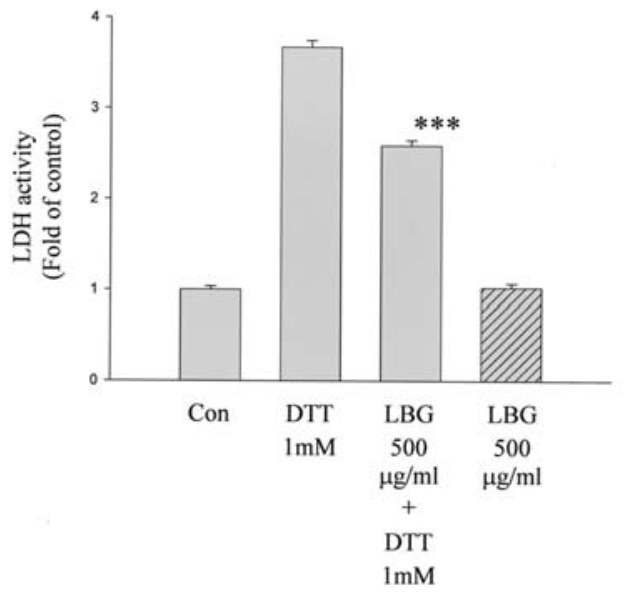

Figure 3. Cytoprotective effects of the LBG extract on DTT-induced cytotoxicity in SH-SY5Y cells. SH-SY5Y cells were treated with LBG (500 $\mu \mathrm{g}$ / $\mathrm{ml}$ ) for $1 \mathrm{~h}$, followed by 24-h incubation with or without DTT (1 mM). LDH activity assay was performed to measure the level of general cytotoxicity. Results are expressed as mean \pm SE from at least three independent experiments. ${ }^{* * *} \mathrm{p}<0.001$ vs. the group treated with DTT only by one-way ANOVA for multiple comparison and Student-Newman-Keuls test as posthoc test.

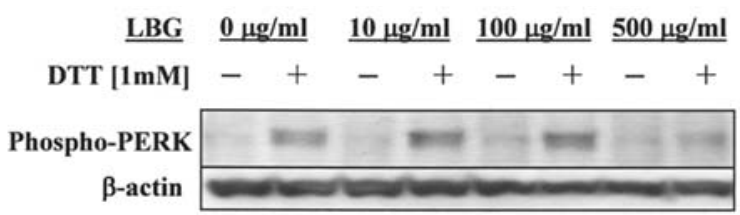

Figure 4. Western blot analysis of phospho-PERK in cultured cortical neurons treated with LBG and DTT. Neurons were treated with different concentrations of LBG for $1 \mathrm{~h}$, followed by 24-h incubation with or without DTT (1 mM). Proteins extracted were subjected to Western blot analysis to detect the level of phospho-PERK. Membranes were stripped to re-probe for $\beta$-actin, which was used as the internal control. The representative blots are from at least three independent experiments.

$1.43 \pm 0.17$-fold of control when compared with the DTT-treated group. The results showed that LBG exerted neuroprotective effects against the reducing stress DTT. The morphology of neurons after different treatments is shown in Fig. 2. In the control group (Fig. 2a), neurons were healthy, with fine neurite network and round cell bodies. Neurons treated with DTT only were dying (Fig. 2b), as revealed by the destructive neuronal network and shrinkage of cell bodies. LBG alone did not alter the morphology of the neurons (Fig. 2c). Pretreatment with LBG protected neurons against DTT toxicity, as shown by the fine morphology in Fig. 2d.

Cytoprotective effects of the LBG extract in SH-SY5Y cells. The cytoprotective effects of LBG were re-confirmed using the human neuroblastoma cell-line, SH-SY5Y. DTT markedly increased the LDH activity to 3.67 \pm 0.07 -fold of control in SH-SY5Y cells (Fig. 3). Pretreatment with LBG at $500 \mu \mathrm{g} /$ $\mathrm{ml}$ for $1 \mathrm{~h}$ significantly reduced the LDH activity to $2.59 \pm 0.06$-fold of control when compared with the group treated with DTT only. The results further proved that LBG exerts cyto-protective effects against the reducing stress DTT in neurons. 
Western blot analysis of phospho-PERK in neurons treated with $L B G$. DTT is one of the ER-stress inducers which can activate the phosphorylation of PERK (18). In order to investigate whether the neuroprotective effects of LBG are related to ER-stress signaling pathways, proteins extracted after treatment were subjected to Western blotting to detect the level of phospho-PERK. Fig. 4 shows that DTT induced the phosphorylated form of PERK. Pretreatment with LBG at $500 \mu \mathrm{g} / \mathrm{ml}$ significantly attenuated the level of phospho-PERK. The protein level of $ß$-actin was unchanged after all treatment.

\section{Discussion}

This is so far the first report showing that the extract from Lycium barbarum can protect neurons from cell death induced by the reducing agent, DTT. We have demonstrated that the extract from L. barbarum, LBG, exhibits cytoprotective effects against the reducing stress in primary cultured cortical neurons by attenuating the LDH activity and the caspase-3like activity triggered by DTT. Pretreatment with LBG in the SH-SY5Y cell line has a similar cytoprotective effect, as shown by the LDH activity assay. DTT is an ER stress inducer which can activate PERK (18). LBG can markedly attenuate the phosphorylation of PERK induced by DTT. Our results suggest that the extract from L. barbarum has novel protective effects in neurons against reducing stress in the ER.

Extracts isolated from $L$. barbarum have been investigated extensively in the past 10 years. The biological effects of L. barbarum include anti-aging, anti-oxidation, anti-tumor and immuno-stimulating (1-5). The anti-oxidative property of $L$. barbarum should be attributed to its rich anti-oxidants such as carotenoids, riboflavin, ascorbic acid, thiamine and nicotinic acid (3). In our previous study (6), we have shown that an aqueous extract from L. barbarum (LBA, mainly consists of polysaccharides) protects neurons from $A \beta$ peptideinduced apoptosis by interrupting the c-Jun N-terminal kinase (JNK)-c-Jun signaling pathway. Since the AB peptide can also induce oxidative stress, it is also possible that LBA exerts neuroprotective effects via anti-oxidation. The present study provides new information about the biological activity of the extract from $L$. barbarum by proving that LBG exhibits cytoprotective effects against the reducing stress triggered by DTT.

In order to investigate the cytoprotective effects of $L$. barbarum against non-oxidative stress, we chose a commonly used reducing agent, DTT, to trigger neuronal cell death. Owing to its reducing capability, DTT is a well-known ER stress inducer (8). The ER oxido-reductive potential is disturbed by DTT, affecting disulfide bond formation, which can result in improper protein folding. Prolonged disruption of protein folding may lead to cell death. A recent study demonstrated that DTT treatment effectively triggered Eca109 cell apoptosis by activating p38 MAP kinase (19). Another report also shows that DTT can induce the PERK-dependent activation of both JNK and p38 MAP kinase (20). Our data show that pretreatment with LBG significantly attenuates DTT-induced PERK phosphorylation. Therefore, it is of interest to investigate whether the signaling pathways of JNK and p38 MAPK are involved in the protective mechanisms of LBG against DTT toxicity in future study.
Collectively, our results suggest that extract from $L$. barbarum possesses cytoprotective effects against reducing stress on the ER. Further studies are needed to elucidate the major active components in L. barbarum responsible for this anti-reducing property.

\section{Acknowledgements}

M.S.Y. and Y.S.H. are supported by postgraduate studentship from The University of Hong Kong. W.H.Y. would like to thank the Department of Chemistry, The University of Hong Kong for support. The study is supported by HKU Strategic Theme Research on Healthy Aging, HKU Technology Transfer Seed Funding, and Area of Excellence (AoE/P-10/01) HKU Seed Funding for Basic Research (200511159072). We also thank Ms. Dorothy Chan and Miss Cora Lai for technical help in the cell culture experiments.

\section{References}

1. Gan L, Hua ZS, Liang YX and Bi XH: Immunomodulation and antitumor activity by a polysaccharide-protein complex from Lycium barbarum. Int Immunopharmacol 4: 563-569, 2004

2. Deng HB, Cui DP, Jiang JM, Feng YC, Cai NS and Li DD: Inhibiting effects of Achyranthes bidentata polysaccharide and Lycium barbarum polysaccharide on non-enzyme glycation in D-galactose induced mouse aging model. Biomed Environ Sci 16: $267-275,2003$.

3. Luo Q, Cai Y, Yan J, Sun M and Corke H: Hypoglycemic and hypolipidemic effects and antioxidant activity of fruit extracts from Lycium barbarum. Life Sci 76: 137-149, 2004.

4. Huang $X$, Yang M, Wu X and Yan J: Study on protective action of Lycium barbarum polysaccharides on DNA impairments of testicle cells in mice. Wei Sheng Yan Jiu 32: 599-601, 2003.

5. Wang Y, Zhao H, Sheng X, Gambino PE, Costello B and Bojanowski K: Protective effect of Fructus Lycii polysaccharides against time and hyperthermia-induced damage in cultured seminiferous epithelium. J Ethnopharmacol 82: 169-175, 2002.

6. Yu MS, Leung SKY, Lai SW, Che CM, Zee SY, So KF, Yuen WH and Chang RCC: Neuroprotective effects of antiaging oriental medicine Lycium barbarum against beta-amyloid peptide neurotoxicity. Exp Gerontol 40: 716-727, 2005.

7. Iuvone $T$, Esposito G, Esposito R, Santamaria R, Di Rosa M and Izzo AA: Neuroprotective effect of cannabidiol, a nonpsychoactive component from Cannabis sativa, on beta-amyloidinduced toxicity in PC12 cells. J Neurochem 89: 134-141, 2004.

8. Gilmore WJ and Kirby GM: Endoplasmic reticulum stress due to altered cellular redox status positively regulates murine hepatic CYP2A5 expression. J Pharmacol Exp Ther 308: 600608, 2004.

9. Trotter EW and Grant CM: Thioredoxins are required for protection against a reductive stress in the yeast Saccharomyces cerevisiae. Mol Microbiol 46: 869-878, 2002.

10. Chang RCC, Suen KC, Ma CH, Elyaman W, Ng HK and Hugon J: Involvement of double-stranded RNA-dependent protein kinase and phosphorylation of eukaryotic initiation factor2alpha in neuronal degeneration. J Neurochem 83: 1215-1225, 2002.

11. Lin KF, Chang RCC, Suen KC, So KF and Hugon J: Modulation of calcium/calmodulin kinase-II provides partial neuroprotection against beta-amyloid peptide toxicity. Eur J Neurosci 19: 2047-2055, 2004.

12. Suen KC, Lin KF, Elyaman W, So KF, Chang RCC and Hugon J: Reduction of calcium release from the endoplasmic reticulum could only provide partial neuroprotection against beta-amyloid peptide toxicity. J Neurochem 87: 1413-1426, 2003.

13. Suen KC, Yu MS, So KF, Chang RCC and Hugon J: Upstream signaling pathways leading to the activation of double-stranded RNA-dependent serine/threonine protein kinase in beta-amyloid peptide neurotoxicity. J Biol Chem 278: 49819-49827, 2003. 
14. Yu MS, Lai SW, Lin KF, Fang JN, Yuen WH and Chang RCC: Characterization of polysaccharides from the flowers of Nerium indicum and their neuroprotective effects. Int J Mol Med 14: 917-924, 2004.

15. Yu MS, Suen KC, Kwok NS, So KF, Hugon J and Chang RCC: Beta-amyloid peptides induce neuronal apoptosis via a mechanism independent of unfolded protein responses. Apoptosis (In press)

16. Fang X, Chang RCC, Yuen WH and Zee SY: Immune modulatory effects of Prunella vulgaris L. Int J Mol Med 15: 491-496, 2005.

17. Fang X, Yu MMS, Yuen WH, Zee SY and Chang RCC: Immune modulatory effects of Prunella vulgaris L. on monocytes/ macrophages. Int J Mol Med 16: 1109-1116, 2005.
18. Bertolotti A, Zhang Y, Hendershot LM, Harding HP and Ron D: Dynamic interaction of $\mathrm{BiP}$ and ER stress transducers in the unfolded-protein response. Nat Cell Biol 2: 326-332, 2000.

19. Zhang QX, Feng R, Zhang W, Ding Y, Yang JY and Liu GH: Role of stress-activated MAP kinase P38 in cisplatin- and DTTinduced apoptosis of the esophageal carcinoma cell line Eca109. World J Gastroenterol 11: 4451-4456, 2005.

20. Liang SH, Zhang W, McGrath BC, Zhang P and Cavener DR: PERK is required to activate the stress-activated MAP kinases and induce the expression of the immediate early genes upon disruption of ER calcium homeostasis. Biochem J 393: 201-209, 2006. 